\title{
Embedded sensor system for five-degree-of-freedom error detection on machine tools
}

\author{
Yubin HUANG ${ }^{1}$, Kuangchao FAN ${ }^{2 *}$, Wei SUN ${ }^{3}$ \\ 1 School of Mechanical Engineering, Dalian University of technology, Dalian, China
}

*Corresponding Author: Kuangchao FAN, No 2, Linggong road, Ganjinzi District, Dalian City, Liaoning Provence, China; fan@dlut. edu.cn

\begin{abstract}
:
Any linear stage of machine tool has inherent six-degree-of-freedom (6-DOF) geometric errors. Its motion control system, however, has only the position feedback. Moreover, the feedback point is not the commanded cutting point. This is the main reason why the positioning error along each axis and the volumetric error in the working space are inevitable. This paper presents a compact 5-DOF sensor system that can be embedded in each axis of motion as additional feedback sensors of the machine tool for the detection of three angular errors and two straightness errors. Using the derived volumetric error model, the feedback point can be transferred to the cutting point. The design principle of the developed 5-DOF sensor system is described. An in-depth study of systematic error compensation due to crosstalk of straightness error and angular error is analyzed. A prototype has been built into a three-axis NC milling machine. The results of a series of the comparison experiments demonstrate the feasibility of the developed sensor system.

Keywords: 5-DOF geometric motion errors; linear axis; measurement; systematic error compensation.
\end{abstract}

\section{Introduction}

The assessment of machine tool's volumetric errors is always an important issue in precision machining. Traditional measurement methods can only use such instruments as laser interferometer, ballbar, etc. to measure the static error of machine tool under the non-working condition ${ }^{[1,2]}$. For the assessment of dynamic errors under cutting condition, the only method is to measure and analyze the cut specimen [3-5]. The direct measurement method of dynamic error is rare.

It is known that the volumetric error of a 3 -axis machine tool is contributed by 21 geometric errors, including 6-DOF geometric errors in each axis and three squareness errors among three axes. In order to fast measure all errors, multidegree-of-freedom simultaneous measurement (MDFM) techniques ${ }^{[6]}$ have been developed in the past three decades. Fan developed a 3-beam 6-DOF measuring system for a linear stage ${ }^{[7]}$ and a 4-beam 6-DOF system for the XY stage ${ }^{[8]}$ based on laser Doppler displacement meters. Liu ${ }^{[9]}$ developed a 3-beam 6-DOF system based on fiber coupled laser interferometer. Feng and Cui ${ }^{[10-13]}$ et al. developed a series of 2-beam 6-DOF measurement system. Huang ${ }^{[17]}$ developed a 2-beam 5-DOF measurement system based on a monolithic prism to generate a pair of parallel laser beam.
Chen developed a 2-beam 6-DOF measurement system based on a straightness interferometer with rotational error compensation. Gillmer and $\mathrm{Yu}{ }^{[14-16]}$ developed a single beam 6-DOF measurement system based on Polarization Optics and wavefront aberration effects.

The applications of MDFM also have received much attentions. $\mathrm{Ni}{ }^{[18,19]}$ developed a series of 3-beam MDFM systems for monitoring errors of the coordinate measurement machine and machine tool. Chen ${ }^{[20]}$ and $\mathrm{Li}$ ${ }^{[21]}$ applied MDFM to simultaneously measure the 6-DOF and 5-DOF errors of a rotary axis, respectively. Commercial MDFM systems are also available on the market, such as XM-60 (Renishaw Inc, UK), XD5 (API Inc, USA) and SP1500 C5 (SIOS Gmbh, Germany) ${ }^{[22]}$.

Although the above-mentioned MDFM systems are useful, however, their costs are high and they are all designed as measuring instrument for quasi-static geometric errors. The MDFM system designed for permanently fixed sensor in each linear axis of the machine tool can not only detect quasi-static errors but also dynamic geometric errors under cutting. This kind of embedded sensor system has not been seen yet.

This paper presents a low-cost and compact 5-DOF measurement system for monitoring all geometric errors except position sensing of the linear stage. A collimated 
laser diode is used as the light source to reduce the system cost. The motivation of developing such an embedded sensor system is explained in section 2. The schematic of systems and the measurement principles of each error are described in section 3 . In section 4 , a series of experiments have been carried out to verify the feasibility of the sensor system.

\section{Multi-sensor feedback NC control system}

Any numerically controlled (NC) precision machine is constructed by some moving stages and a functional head. The stages can be composed of linear stages and rotary stages to form three- to multi-axis machines. The functional head characterizes the machine capability, such as the spindle head of a machine tool, the lithography head of a wafer stepper, the bonding head of an IC die bonder, the probe head of a measuring machine, etc. As it is called a precision machine, its moving position must be precisely controlled. The typical position control loop of machine tool can be seen in Fig. 1. It is noted that the commanded position is the tool point, but the feedback position is the sensing point. If the moving stage has a tilted angle at this position, the actual position of tool (X', functional point) is deviated from the sensing point $(\mathrm{X})$ by $\Delta \mathrm{X}$, which is called the positioning error and is the tilted angle multiplied by the offset L. Such a positioning error is caused by the Abbe principle because the measuring axis is not in line with the functional axis ${ }^{[25]}$. In fact, each moving stage has all 6-DOF geometric errors, as shown in Fig. 2. The actual positional error in the working space is called volumetric error which is caused by related linear errors, angular errors and squareness errors induced positional errors at the functional point. Modelling the volumetric errors is dependent on the configuration of the machine tool, normally by the technique of Homogeneous Transformation Matrix (HTM) ${ }^{[1,2]}$. Therefore, in order to compensate the volumetric error during cutting, a 5-DOF sensor system must be equipped in the machine tool and establishes a 6-DOF feedback control loop of NC machine tools, as schematically shown in Fig. 3. This is the main reason why the embedded 5-DOF sensor system is urgently needed in precision machine tools.

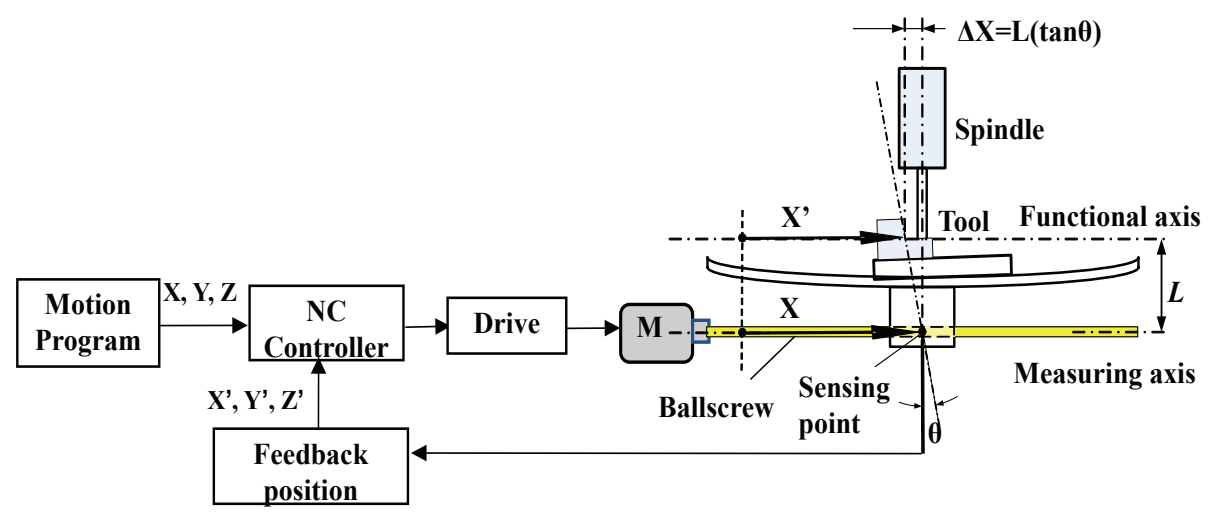

Figure 1. Typical control loop of NC machine tool

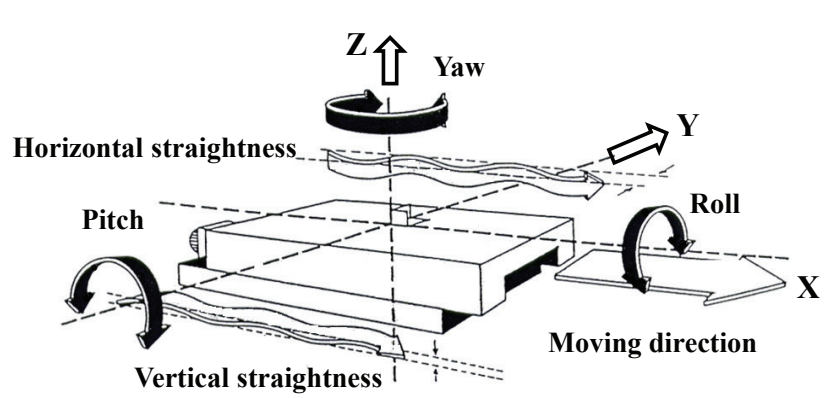

Figure 2. 6-DOF geometric errors of the linear stage

\section{Design of a 5-DOF sensor system}

The schematic diagram of the proposed 5-DOF sensor system is shown in Fig. 4(a). It is an extended work from our previous 4-DOF sensor system ${ }^{[26]}$ with the addition of roll error sensing. The stationary part is fixed on the base of the linear stage and the moving part is moved with the motion table.

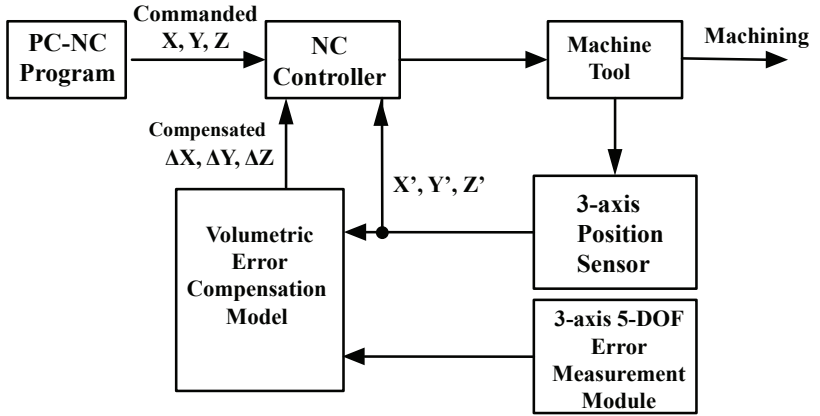

Figure 3. The proposed feedback control loop with 6-DOF sensors

In the stationary part, the laser beam from the laser diode (LD) is reflected by a mirror M1 and is then split by a polarized beam splitter (PBS) into 2 beams. The transmitted beam is used to measure straightness, yaw and pitch errors of the moving table by the quadrant photodetector (QPD3) and the autocollimator unit of focus lens (FL2) and QPD2, respectively. The reflected beam from PBS is spilt by a beam splitter (BS1), from which the reflected beam enters 
to an autocollimator unit consisting of a focus lens (FL1) and a (QPD1) to measure the laser drift angle. The other transmitted beam turns to a outgoing beam parallel to the first beam after deflected by M2. A QPD4 detects the straightness error motion of the moving table at this point. Comparing with the vertical straightness error detected at QPD3, the difference reveals the roll error of the moving table. There are two PZT actuators embedded in the mechanism of angle steering mirror (M1) for real-time

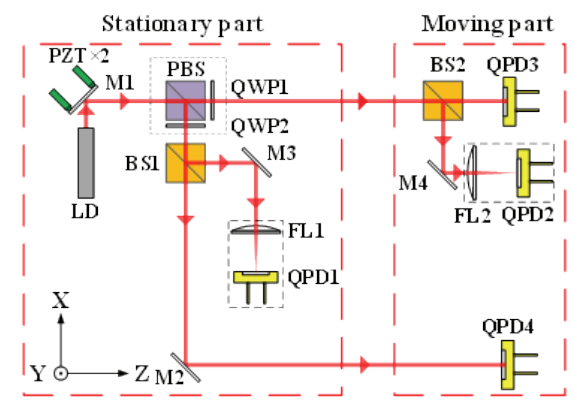

(a) compensation of the drifted angle of the LD. The detailed design and control of this fine-tuning mirror can be seen in our previous 4 -DOF sensor system ${ }^{[26]}$. Fig. 4(b) shows the photo of the prototype of this 5 -DOF sensor. When installing such a sensor system in the machine tool, the optical path is covered by a bellow shield so that the cutting chips, fluid and air disturbance can be avoided, as shown in Fig. 5. All three axes of the machine tool are treated in the same way.

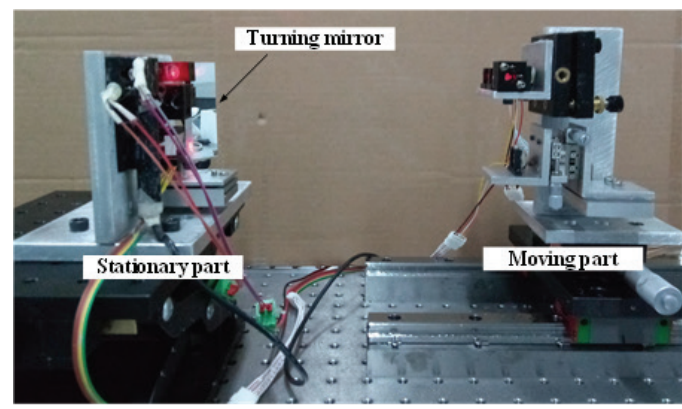

(b)

Figure 4. Schematic of the development 5-DOF measurement system: (a) optical system, (b) prototype of sensor system

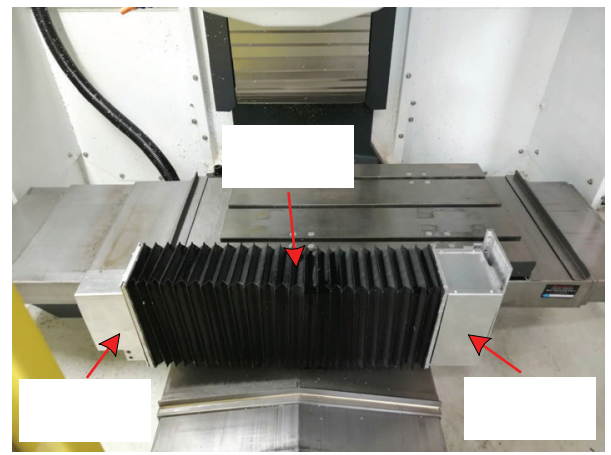

Figure 5. Mounting the 5-DOF sensor system in one axis of the machine tool

\subsection{Measurement principle}

It can be seen in Fig. 4(a) that the upper part of optical system is a 4 -DOF sensor system ${ }^{[26]}$ that detects the vertical and horizontal straightness errors, and the pitch and yaw errors, of the moving table. The center of QPD3 is defined as the point of straightness error measurement. This point is also the feedback point of measured straightness errors to the controller as illustrated in Fig. 3. Changing to different feedback point will result in different straightness error due to rigid body kinematics. Therefore, this point on the machine tool must be recorded. The corresponding straightness errors detected by the QPD3 can be expressed by:

$$
\begin{aligned}
& \delta_{x}=\Delta x_{Q P D 3}=k_{\delta x} \frac{\left(i_{1}+i_{4}\right)-\left(i_{2}+i_{3}\right)}{\left(i_{1}+i_{2}+i_{3}+i_{4}\right)} \\
& \delta_{y}=\Delta y_{Q P D 3}=k_{\delta y} \frac{\left(i_{1}+i_{2}\right)-\left(i_{3}+i_{4}\right)}{\left(i_{1}+i_{2}+i_{3}+i_{4}\right)}
\end{aligned}
$$

Where, $\delta x$ and $\delta y$ are the straightness errors of the measured linear stage in the $\mathrm{X}$ and $\mathrm{Y}$ directions, respectively. $\Delta x_{Q P D 3}$ and $\Delta_{y Q P D 3}$ are the corresponding spot movement on QPD3 respectively in the $\mathrm{X}$ and $\mathrm{Y}$ directions, $i_{1}, i_{2}, i_{3}, i_{4}$ indicate the output currents acquired by the QPD's $1 \sim 4$ quadrants, and $k_{\delta x}, k_{\delta y}$ are constants obtained by the calibration experiment.

The yaw and pitch errors measured by the autocollimator unit of FL2 and QPD2 can be expressed by

$\varepsilon_{x}=\frac{\Delta x_{Q P D 2}}{f}$ and $\quad \varepsilon_{y}=\frac{\Delta y_{Q P D 2}}{f}$

where, $\varepsilon_{y}$ and $\varepsilon_{x}$ are yaw and Pitch errors of the measurement linear stage, respectively, $\Delta x_{Q P D 2}$ and $\Delta y_{Q P D 2}$ are the corresponding outputs of QPD2, and $f$ is the focal length of FL2.

The principle of roll measurement used in this system is a typical dual parallel beam measurement method. Fig. 6 shows a simplified diagram from Fig. 4(a). The roll error of the moving stage can be obtained by the following formula: 
$\varepsilon_{z}=\frac{\left(\delta x-\delta x_{4}\right)}{L}$

where, $\delta x$ is the straightness error in $\mathrm{x}$-direction measured by QPD3, $\delta x_{4}$ is the beam spot movement in $\mathrm{x}$-direction measured by QPD4, L is the distance between the center of QPD3 and the center of QPD4 in the vertical direction.

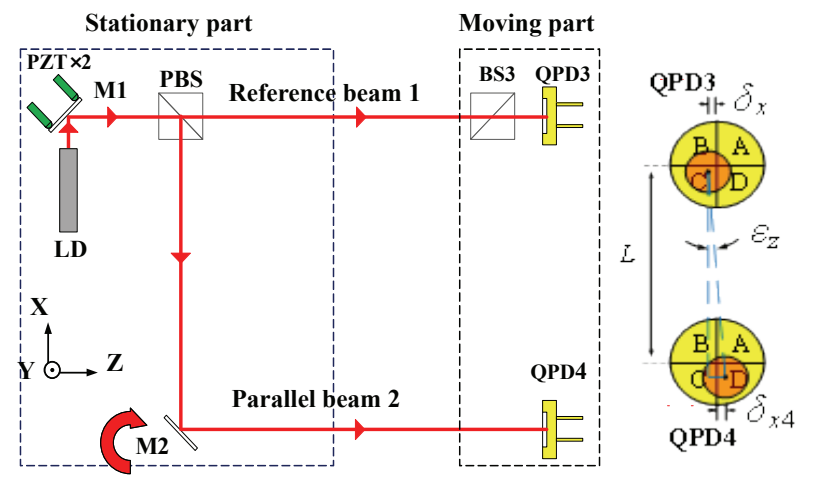

Figure 6. Measurement principle of the roll error

\subsection{Feedback compensation for laser beam drift}

In order to eliminate the measurement error caused by the laser beam drifts, a beam drift feedback control module proposed before ${ }^{[23]}$ is integrated into the system. The angle measurement unit consisted of FL1 and QPD1 is used to measure the angular drift of the emergent laser beam. A fine-tuning mirror driven by two PZT actuators is used as the drift feedback control mechanism to make the angle of the emerging laser beam stabilized through the PID control. To suppress the spot parallel drift caused by the change of the reflection point on the steering mirror ${ }^{[28]}$, we optimized the structural design to ensure that total linear drift is within $\pm 2.56 \mu \mathrm{m}$ within the full motion of the PZTs.

\section{Error compensation in angular error mea- surement}

The measurement error compensation is a serious problem for the MDFM system, especially in the small size, highly compact measurement devices. Except the conventional crosstalk error due to the assembly error between the QPD's coordinate system and the actual coordinate system of the stage, there still have some error sources which would significantly affect the accuracy of angular error measurement.

\section{1 systematic error in yaw and pitch measurement}

In the proposed 5-DOF measurement system, the laser incident position of FL2 would change if the straightness error exists. As shown solid lines in Fig. 7(a), In the ideal condition, the incident position of the laser has no influence on the angular measurement since the QPD2 is set on the focus plane of FL2. In actual condition, however, due to the assembly error, the change of laser's incident position would cause a positioning shift of the focused beam spot on the QP2 surface, as shown in Fig. 7(b).

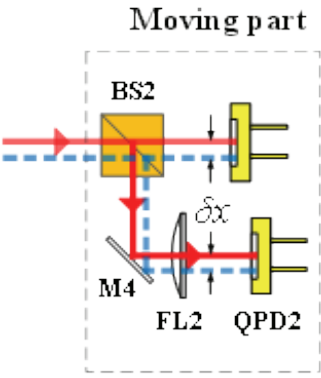

(a)

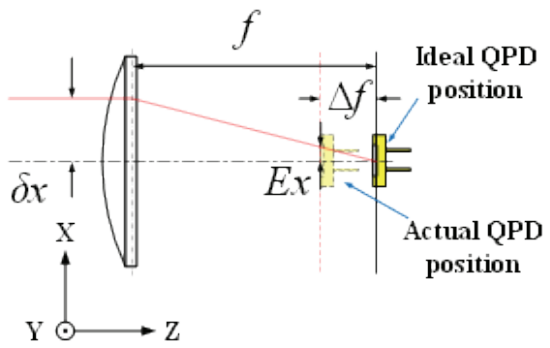

(b)

Figure 7. Systematic error of yaw and pitch angle measurement caused by the straightness error: (a) the laser's incident position shift due to the straightness error, (b) position shift of focused beam spot on the QPD surface due to the change of laser's incident position.

The relationship between the straightness error, the assembly error of QPD2's position and the position shift of the focused beam spot can be express as follows:

$$
E x=\delta x(\Delta f / f) \text { and } E y=\delta y(\Delta f / f)
$$

where, $\delta x$ and $\delta y$ are the straightness errors of the measured linear stage in the $\mathrm{X}$ and $\mathrm{Y}$ directions, respectively. $E x$ and Ey are the position shifts of the focused beam spot on the QPD2 surface, respectively. $f$ is the focal length of FL2 and $\Delta f$ is the offset distance between QPD2 surface and the focus plane of FL2 caused by assembly error. The extra shift of focused beam spot would cause a systematic measurement error $E_{\varepsilon x}$ in yaw error and $E_{\varepsilon y}$ in pitch error as

$$
\begin{aligned}
& E_{\varepsilon x}=\frac{E_{x}}{f}=\delta_{x} \frac{\Delta f}{f^{2}} \text { and } \quad E_{\varepsilon y}=\frac{E_{y}}{f}=\delta_{y} \frac{\Delta f}{f^{2}}
\end{aligned}
$$

In order to reduce the size of the 5-DOFsensor system, the focus length of FL2 is limit, which leads to a result that the measurement error caused by the change of incident position cannot be ignored. Hence, it is necessary to calibrate and compensate for these errors in the measurement software. The calibration test set-up is shown in Fig. 8(a). The incident position was measured by QPD3 of the 5-DOF system, and the autocollimator (made by 
AutoMAT Co. model 5000U) was used to detect the angular error of moving stage. The calibrated results are shown in Fig. 9. It is clearly seen that the relationship between incident position change and the angular measurement error is quite linear. Due to the influence of the gap between each quadrant of QPDs, the assembly error of QPD sensing surface needs to be limited to $\pm 1.5 \mathrm{~mm}$ of focal length of the FL2. When the assembly error is too large, both the autocollimator measurement principle and the defocus error compensation formula will not work properly.

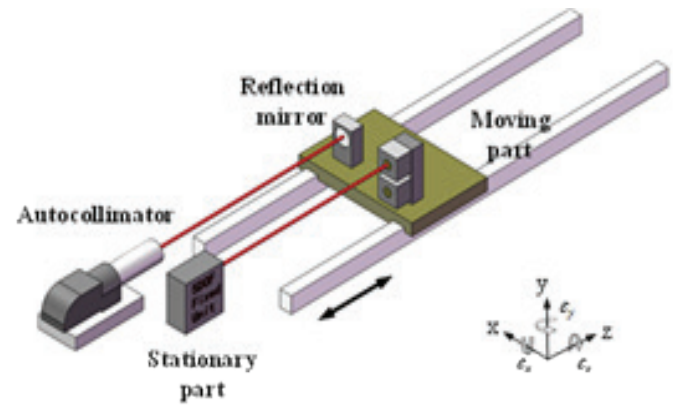

(a)
In order to verify the effectiveness of error compensation, the readings of 5-DOF's measured yaw and pitch were compared to the commercial autocollimator. The comparison test is shown in Fig. 8(b) and the comparison results are shown in Fig. 10 and Fig. 11. It is seen that the residuals after compensation have been significantly reduced from about 15 arcsec to about 2 arcsec in yaw error and from about 4 arcsec to about 1.5 arcsec in pitch error. The trend of the residual curves is similar to the straightness error which will be presented in Section 5.

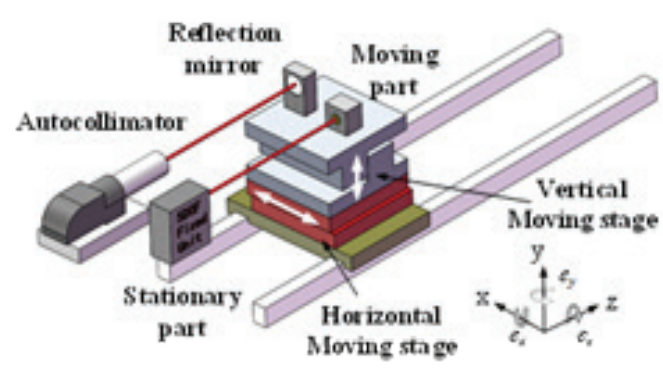

(b)

Figure 8. Experiment set-up of the yaw and pitch systematic measurement error tests. (a) Calibration set-up, (b) Comparison set-up

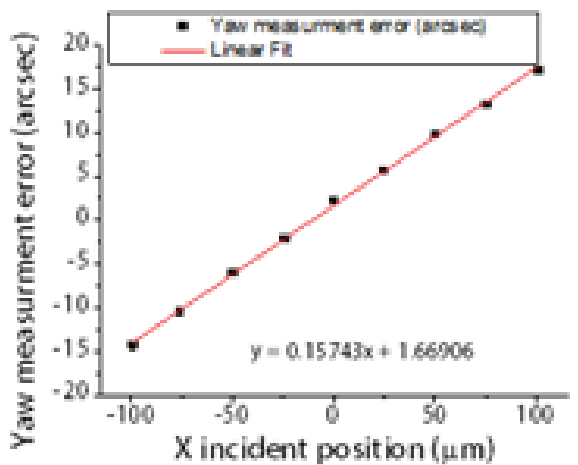

(a)

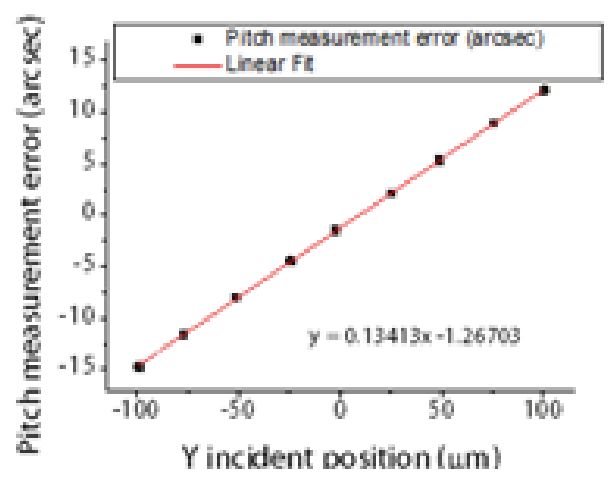

(b)

Figure 9. Calibration results of the relationship between the incident positon and angular measurement error: (a) Yaw, (b) Pitch.

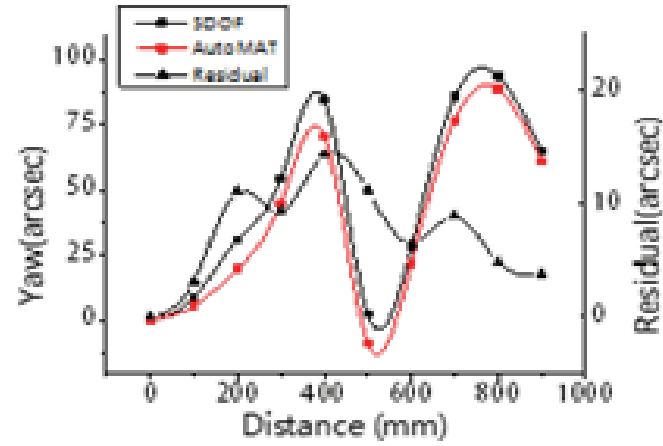

(a)

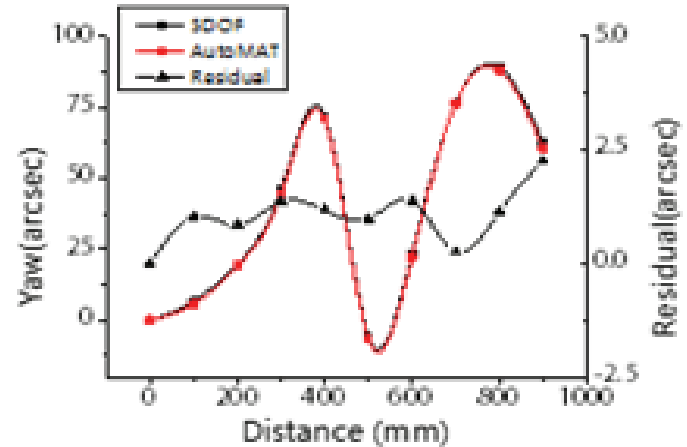

(b)

Figure 10. Systematic error of yaw angle measurement: (a) before compensation, (b) after compensation 


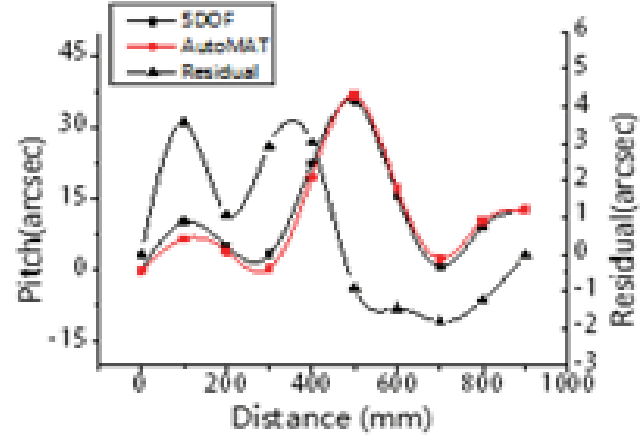

(a)

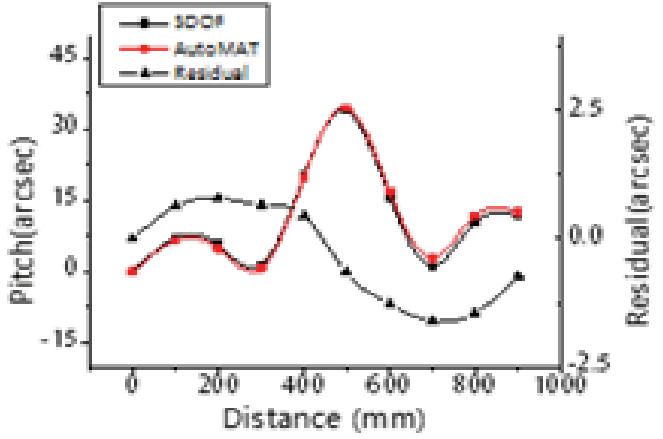

(b)

Figure 11. Systematic error of pitch angle measurement: (a) before compensation, (b) after compensation

\subsection{Systematic error in roll measurement}

The measurement accuracy of the principle of roll angle based on the method of dual parallel beams is significantly affected by the parallelism between the two referenced beams. If the two beams are not parallel with a small angle, a measurement error of the roll would increase with the measured distance. Therefore, an error correction model is required to separate the actual roll error from the non-parallelism induced error. This part has been comprehensively considered and solved in our previous work on the robust roll error measurement study ${ }^{[27]}$. The technical detail is not repeated here.

\section{Experiment results and discussions}

\subsection{Calibration experiments}

The straightness errors were calibrated by a Laser Doppler
Displacement Meter (LDDM, made by Optodyne Inc. USA, model LICS-100) and the angular errors of pitch and yaw were calibrated by an autocollimator. The roll error was calibrated by an electronic level.

The calibration results of straightness measurements are shown in Fig. 12 (a) and (b). Within a measurement range of $\pm 100 \mu \mathrm{m}$. The accuracy in each direction is within $\pm 0.5 \mu \mathrm{m}$. The calibration results of yaw and pitch measurements are shown in Fig. 12 (c) and (d). The measurement range is \pm 100 arcsec. The accuracy is within \pm 0.5 arcsec in either pitch or yaw error. The calibration result of roll measurement is shown in Fig. 12(e). The measurement range is \pm 100 arcsec. The accuracy is also within \pm 0.5 arcsec. These performances verify the feasibility of the developed 5-DOF sensor system.

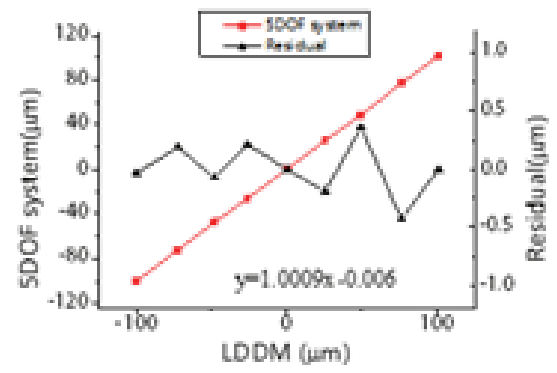

(a)

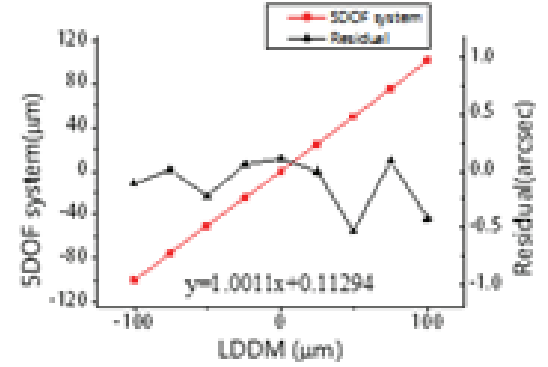

(b)

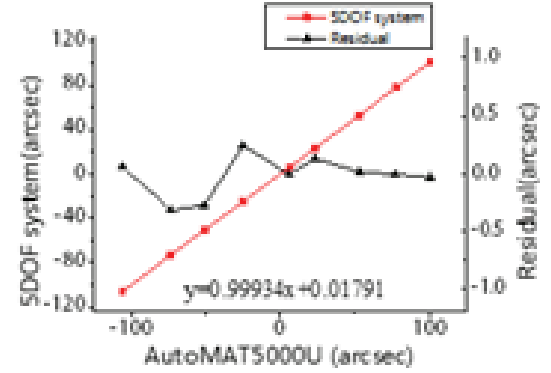

(c)

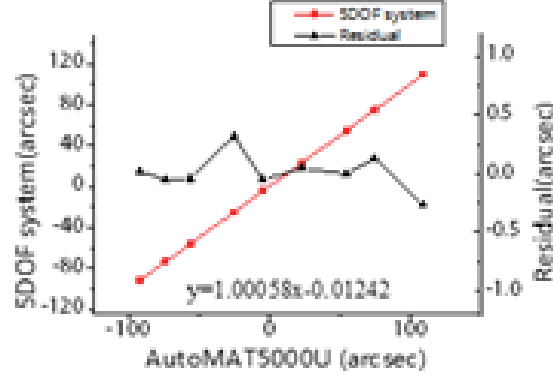

(a)

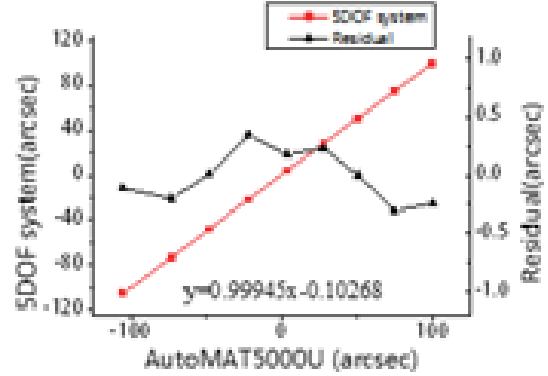

(b)

Figure 12. Results of calibration tests: (a) horizontal straightness, (b) vertical straightness, (c) yaw, (d) pitch, (e) roll. 


\subsection{Comparison and repeatability tests}

A series of comparison experiments were made to demonstrate the effectiveness of this 5-DOF sensor system. The measurement results obtained by this sensor system are compared with the measurement results by the commercial measuring instrument. The measured distance was $900 \mathrm{~mm}$ with a step size of $100 \mathrm{~mm}$. Each comparison was repeated 5 times.

First of all the comparison experiment set-up for straightness error measurement is shown in Fig. 13(a). The straightness measurement was compared with a commercial Laser Straightness Measuring System (LSMS made by 3 DFamily, Taiwan, accuracy \pm 0.5 arcsec). An electronic level (made by Qianshao Co. China, model WL2 , accuracy \pm 0.5 arcsec) was used to measure the roll angle in order to compensate the Bryan error in straightness measurement. According to the Bryan principle, the straightness error measured at point $A$ is different from that measured at point B, as shown in Fig. 13(b), and it is necessary to transform the straightness measured by the LSMS at point $\mathrm{B}$ to the point $\mathrm{A}$ for comparison. The angular induced error is corrected in association with the offset ${ }^{[25]}$ by the Bryan errors, expressed by:

$\delta_{A y}(z)=\delta_{B y}(z)+\varepsilon_{z}(z) L_{B A x}(z)$

$\delta_{A x}(z)=\delta_{B x}(z)-\varepsilon_{z}(z) L_{B A y}(z)$

The comparison results of straightness errors are given in Fig. 14(a) to (c) for the horizontal straightness and (d) to (f) for the vertical straightness of the tested linear stage. It is clearly seen that not only the trend but also the magnitude, measured errors by two systems are very coincident. The relative errors in both directions are all within $\pm 1.5 \mu \mathrm{m}$.

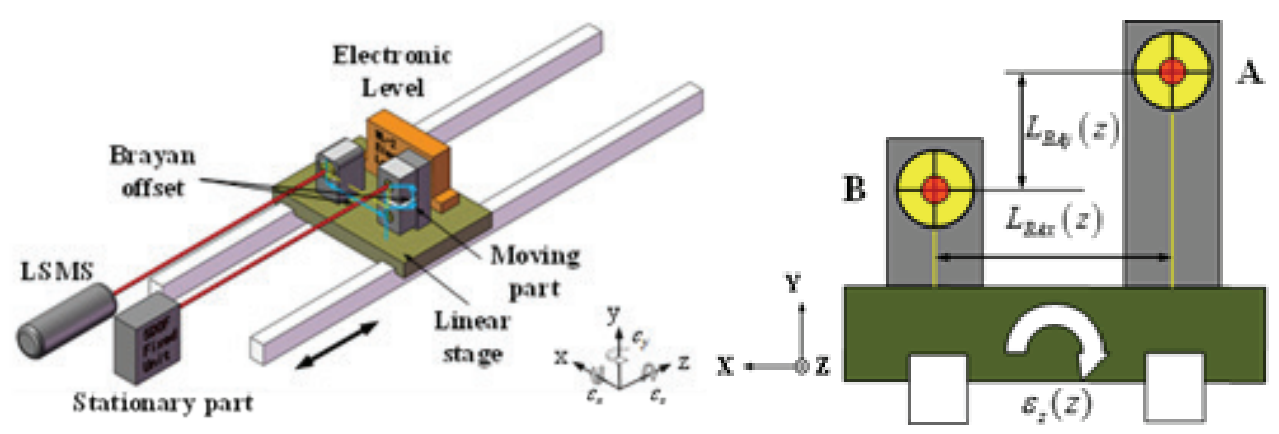

(a)

(b)

Figure 13. Comparison of straightness error measurements (a) experimental set-up, (b) Bryan error correction for different points of measurement

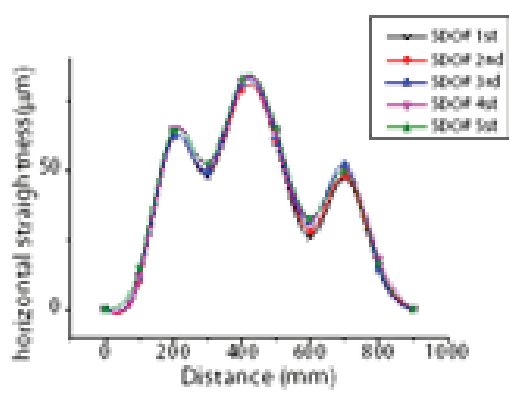

(a)

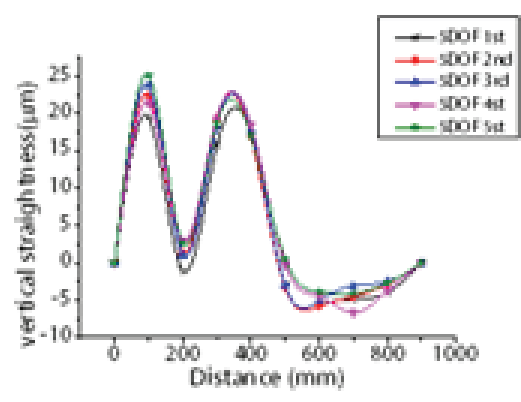

(d)

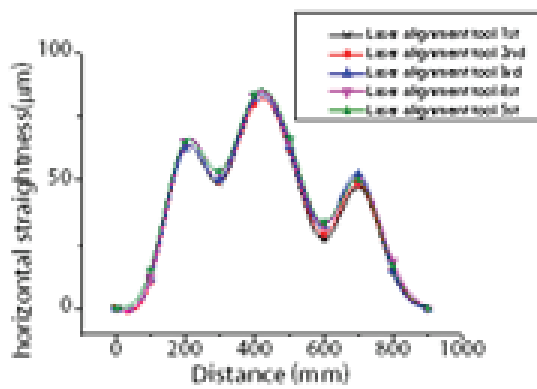

(b)

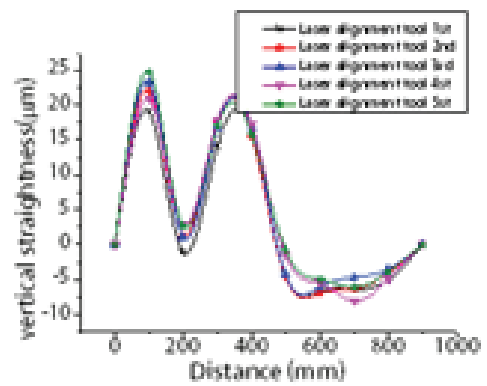

(e)

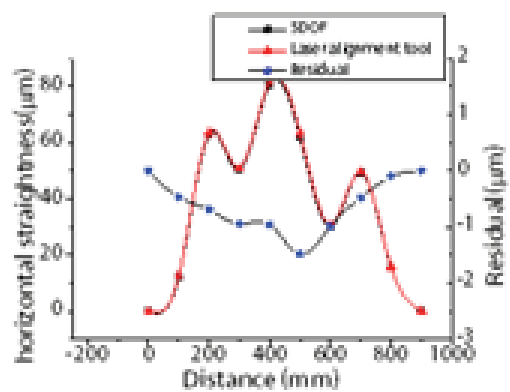

(c)

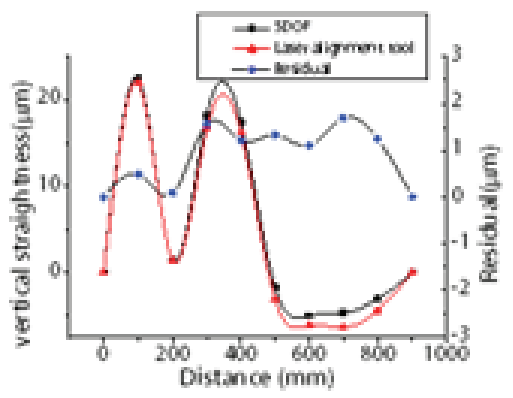

(f)

Figure 14. Straightness repeatability and comparison results: Horizontal straightness: (a) 5-DOF, (b) LSMS, (c) comparison results. Vertical straightness: (d) 5-DOF, (e) laser alignment tool, (f) comparison results. 
The comparison experiment set-up for yaw and pitch error measurement has been shown in Fig. 8(b), and the comparison experiment set-up for roll error is depicted by Fig. 13(a) but without the LSMS. Fig. 15(a) to (c) show results of yaw error measurements, (d) to (f) show results of pitch error measurements, and ( $\mathrm{g}$ ) to (i) show the results of roll error measurements. All are very satisfied. This comparison experiment can be reproduced on any eligible linear motion stage with a stroke of less than $1000 \mathrm{~mm}$ and all 5 geometry errors are within the measurement range of the proposed 5-DOF sensing system.

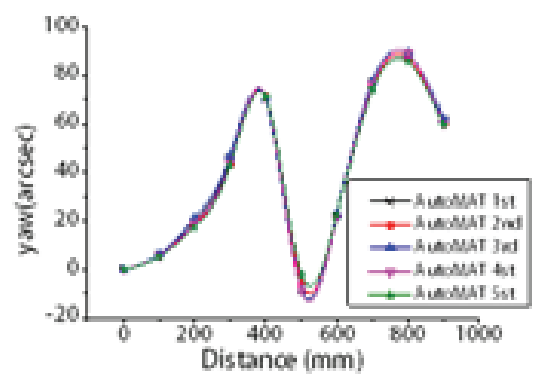

(a)

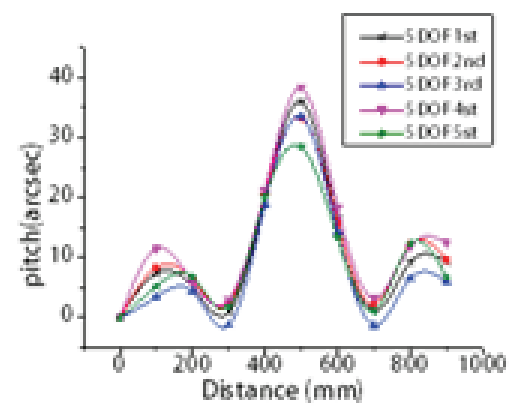

(d)

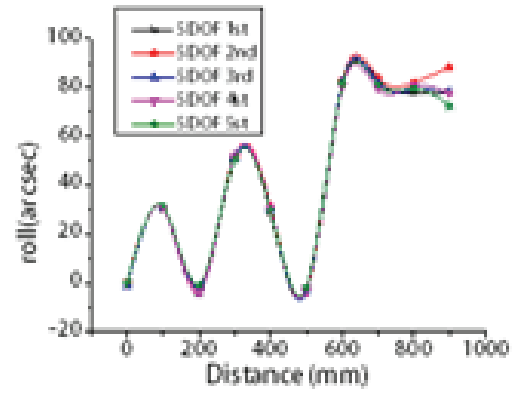

(g)

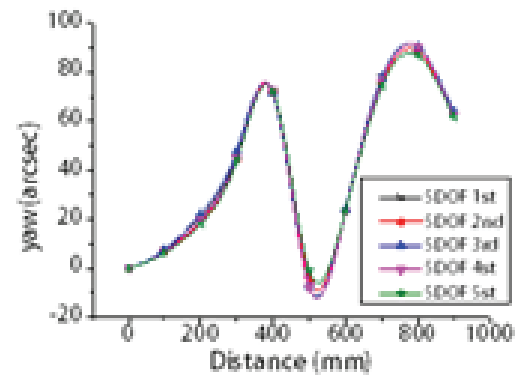

(b)

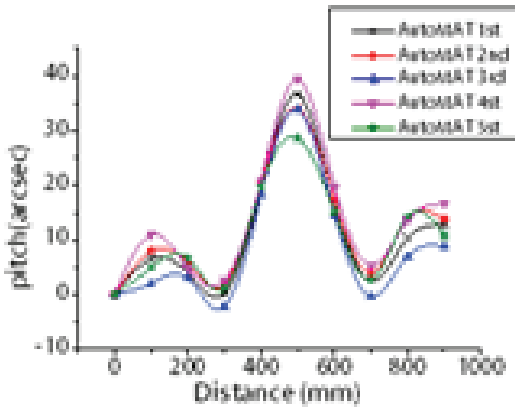

(e)

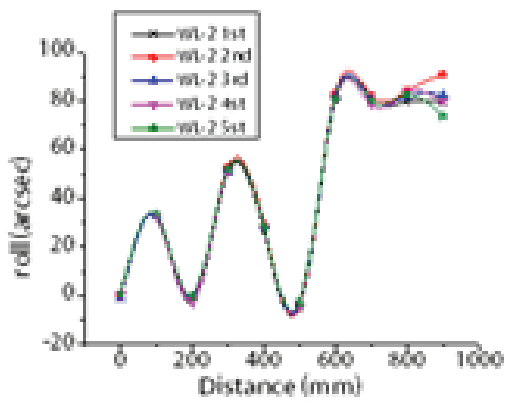

(h)

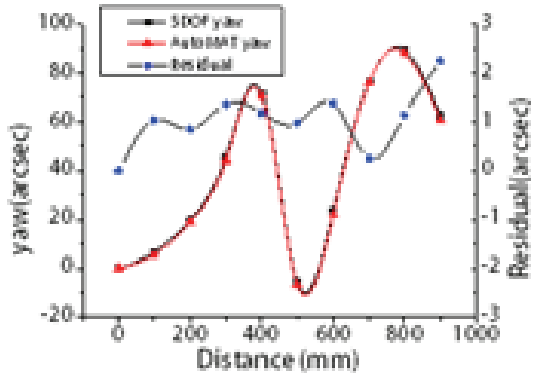

(c)

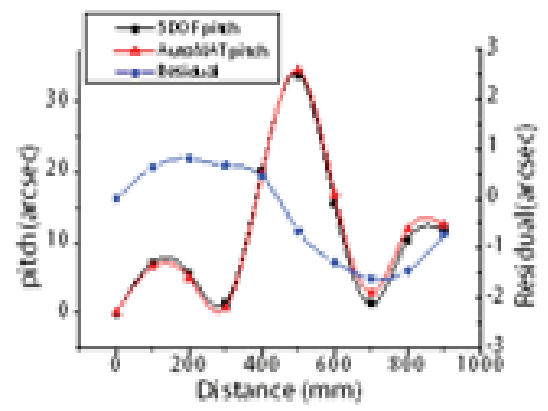

(f)

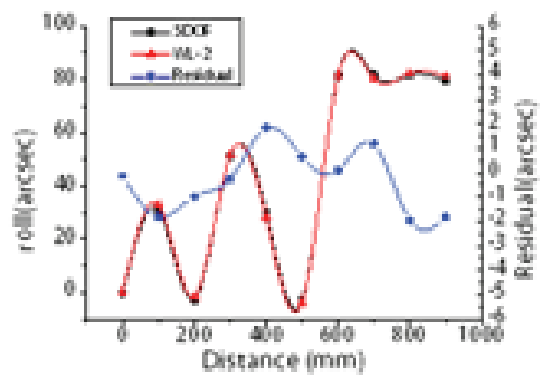

(i)

Figure 15. Angular measurement repeatability and comparison results: Yaw: (a) 5-DOF, (b) autocollimator, (c) comparison results. Pitch: (d) 5-DOF, (e) autocollimator, (f) comparison results. Roll: (g) 5-DOF, (h) electronic level, (i) comparison results.

\subsection{Stability tests}

The stability tests were performed under a general industrial environment without temperature control. The distance between the stationary part and moving part of the 5-DOF system remained $450 \mathrm{~mm}$ and the beam drift error is compensated by the feedback control in real-time. The readings of the 5-DOF system were automatically recorded at each 10 seconds and the total experiment time elapsed 5 hours.
The experimental results shown in Fig. 16 confirm the signal drift of the proposed 5-DOF system is less than 1 $\mu \mathrm{m}$ in straightness measurement, less than one arcsec in yaw and pitch measurement, and less than 2 arcsec in roll measurement, all are in 5 hours. These results prove the stability and feasibility of using this 5-DOF sensor system to detect motion errors of the linear stage of the machine tool. 


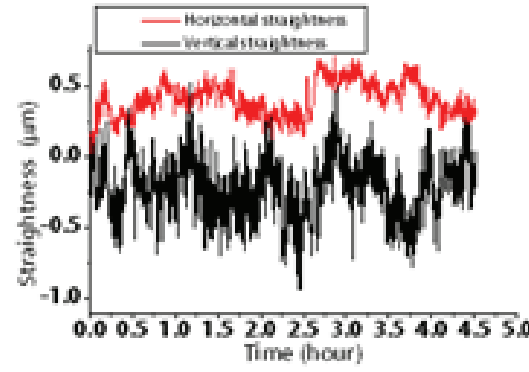

(a)

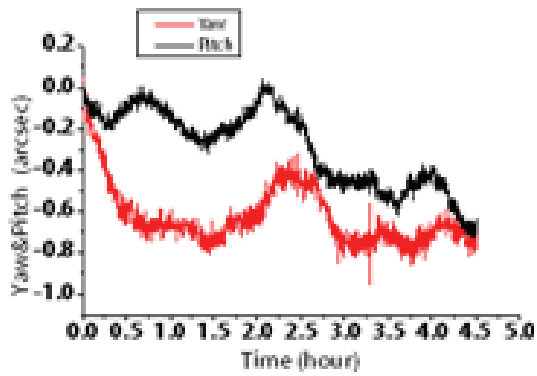

(b)

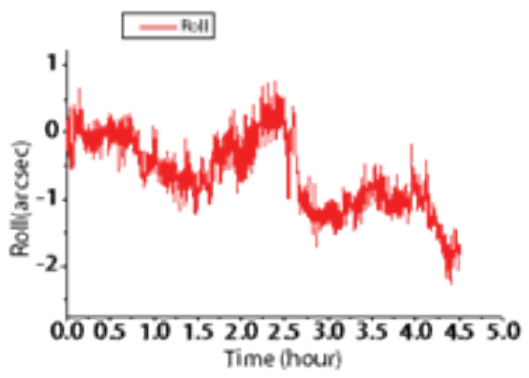

(c)

Figure 16. Results of stability experiments. (a) straightness (b) yaw\&pitch (c) roll

\section{Conclusions}

In this paper, a 5-DOF sensor system for detecting geometric errors of each linear axis of machine tool is proposed. The system is compact in size, simple in optical configuration and low cost. It is to be installed in the machine tool as an embedded sensor to real-time detect 5-DOF geometric errors in each axis of motion. Experimental results show the feasibility and stability of the developed sensor system. It has a great potential to discover the dynamic error of the machine tool during real-cutting condition. It can also extend the current NC control loop from a single sensor feedback system to all 6 sensors feedback system so that the volumetric error compensation is possible. These are all our future works.

Author Contributions: Yubin HUANG carried out all experiments, Kuang-Chao Fan was the principal investigator of the project, and Wei Sun provided laboratory facilities.

Acknowledgement: This research was supported by the fund of The National Key Research and Development Program of China (2017YFF0204800)

\section{References}

[1] Schwenke H, Knapp W, Haitjema H, et al. Geometric error measurement and compensation of machinesan update[J]. CIRP Annals, 2008, 57(2): 660-675.

[2] Ibaraki S, Knapp W. Indirect measurement of volumetric accuracy for three-axis and five-axis machine tools: a review[J]. International Journal of Automation Technology, 2012, 6(2): 110-124.

[3] Ibaraki S, Sawada M, Matsubara A, et al. Machining tests to identify kinematic errors on five-axis machine tools[J]. Precision Engineering, 2010, 34(3): 387-398.

[4] Ibaraki S, Ota Y. A machining test to evaluate geometric errors of five-axis machine tools with its application to thermal deformation test[J]. Procedia CIRP, 2014, 14: 323-328.

[5] Su Z, Wang L. Latest development of a new standard for the testing of five-axis machine tools using an
S-shaped test piece[J]. Proceedings of the Institution of Mechanical Engineers, Part B: Journal of Engineering Manufacture, 2015, 229(7): 1221-1228.

[6] Fan K C, Wang H Y, Yang H W, et al. Techniques of multidegree-of-freedom measurement on the linear motion errors of precision machines[J]. Advanced Optical Technologies, 2014, 3(4): 375-386.

[7] Fan K C, Chen M J, Huang W M. A six-degree-of-freedom measurement system for the motion accuracy of linear stages[J]. International Journal of Machine Tools and Manufacture, 1998, 38(3): 155-164.

[8] Fan K C, Chen M J. A 6-degree-of-freedom measurement system for the accuracy of $X Y$ stages[J]. Precision Engineering, 2000, 24(1): 15-23.

[9] Liu C H, Jywe W Y, Hsu C C, et al. Development of a laserbased high-precision six-degrees-of-freedom motion errors measuring system for linear stage[J]. Review of scientific instruments, 2005, 76(5): 055110.

[10] Feng Q, Zhang B, Cui C, et al. Development of a simple system for simultaneously measuring 6DOF geometric motion errors of a linear guide[J]. Optics express, 2013, 21(22): 25805-25819.

[11] Cui C, Feng Q, Zhang B, et al. System for simultaneously measuring 6DOF geometric motion errors using a polarization maintaining fiber-coupled dual-frequency laser[J]. Optics express, 2016, 24(6): 6735-6748.

[12] Gao S, Zhang B, Feng Q, et al. Errors crosstalk analysis and compensation in the simultaneous measuring system for five-degree-of-freedom geometric error[J]. Applied Optics, 2015, 54(3): 458-466.

[13] Zhao $Y$, Zhang B, Feng Q. Measurement system and model for simultaneously measuring 6DOF geometric errors[J]. Optics express, 2017, 25(18): 20993-21007.

[14] Gillmer S R, Yu X, Wang C, et al. Robust high-dynamicrange optical roll sensing[J]. Optics letters, 2015, 40(11): 2497-2500.

[15] Yu X, Gillmer S R, Ellis J D. Beam geometry, alignment, and wavefront aberration effects on interferometric differential wavefront sensing[J]. Measurement Science and Technology, 2015, 26(12): $125203 .$.

[16] Yu X, Gillmer S R, Woody S C, et al. Development 
of a compact, fiber-coupled, six degree-of-freedom measurement system for precision linear stage metrology[J]. Review of Scientific Instruments, 2016, 87(6): 065109.

[17] Huang P, Li $Y$, Wei $H$, et al. Five-degrees-of-freedom measurement system based on a monolithic prism and phase-sensitive detection technique[J]. Applied optics, 2013, 52(26): 6607-6615.

[18] Wu S M. An on-line measurement technique for machine volumetric error compensation[J]. Ann Arbor, 1993, 1050: 48109.

[19] Huang P S, Ni J. On-line error compensation of coordinate measuring machines[J]. International Journal of Machine Tools and Manufacture, 1995, 35(5): 725-738.

[20] Chen B, Xu B, Yan L, et al. Laser straightness interferometer system with rotational error compensation and simultaneous measurement of six degrees of freedom error parameters[J]. Optics express, 2015, 23(7): 90529073.

[21] Li J, Feng Q, Bao C, et al. Method for simultaneous measurement of five DOF motion errors of a rotary axis using a single-mode fiber-coupled laser[J]. Optics express, 2018, 26(3): 2535-2545.
[22] Mutilba U, Gomez-Acedo E, Kortaberria G, et al. Traceability of on-machine tool measurement: a review[J]. Sensors, 2017, 17(7): 1605.

[23] Liu S, Zhang S, Huang $Y$, et al. The Method for Restraining Laser Drift Based on Controlling Mirror[J]. Nanomanufacturing and Metrology, 2018: 1-8.

[24] Torng J, Wang C H, Huang Z N, et al. A novel dualaxis optoelectronic level with refraction principle[J]. Measurement Science and Technology, 2013, 24(3): 035902.

[25] Bryan J B. The Abbe principle revisited: an updated interpretation[J]. Precision Engineering, 1979, 1(3): 129132.

[26] Huang Y B, Fan K C, Sun W, Liu S J. Low cost, compact 4-DOF measurement system with active compensation of beam angular drift error. Optics Express, 2018, 26(13): 17185-17198.

[27] Cai Y, Yang B H, Fan K C. A robust roll angular error measurement method for precision machines. Optics Express, 2019, 27(6): 8027-8036.

[28] Liu S, Tan S, Huang Y, et al. Design of a compact four degree-of-freedom active compensation system to restrain laser's angular drift and parallel drift. Review of Scientific Instruments, 2019, 90(11): 115002. 